\section{The Plight of Patients with Lupus Nephritis during the Outbreak of COVID-19 in Wuhan, China}

To the Editor:

The number of cases of SARS-CoV-2 infection is rising rapidly globally. Severe disease and high fatality are observed in older patients and those with comorbid conditions ${ }^{1}$. However, little is known about patients with rheumatic disease in the epidemic areas. Patients with rheumatic diseases are subject to societal lockdown and enforced quarantine, leading to inaccessibility to healthcare. Also, their immunosuppressed state heightens the fear of contracting coronavirus disease 2019 (COVID-19). Here we report the outcomes of a cohort of 101 patients with lupus nephritis (LN) including 2 confirmed COVID-19 cases during a surge of the outbreak of COVID-19 from January to February 2020, in Wuhan, China.

We conducted a study by questionnaires and telephone interviews in March 2020. Questionnaires were sent to 160 patients with LN followed up in the Department of Nephrology in Renmin Hospital of Wuhan University, China. The questionnaire included age, sex, duration of disease, the classification of LN, followup, use of medications, relapse of disease, quarantine, and infection of COVID-19. The study was approved by the Ethics Committee of Renmin Hospital of Wuhan University (WDRY2020-K149) and informed consent was obtained from patients.

There were 101 responses from questionnaires and telephone interviews. The median age of respondents was 42 years, of which $88 \%(89 / 101)$ were female. The number of LN classification I, II, III, III/V, IV, IV/V, V and unknown was $6,3,6,2,12,11,13$, and 48 , respectively. Sixty percent $(61 / 101)$ were not able to attend the rheumatology outpatient appointments because of the close of the clinic and the shutdown of transportation during the sudden unprecedented lockdown in Wuhan, and 13\% (13/101) had to consult physicians online. Twenty-five percent $(25 / 101)$ of the patients discontinued medications (Table 1), of whom 13 did so owing to limited access to healthcare. Five patients (5\%) experienced disease flare.

Five patients were quarantined and tested for COVID-19. Two patients were confirmed to have contracted COVID-19. Both had mild symptoms and did not require supplemental oxygen. Patient A was a 23-year-old female with class IV LN diagnosed 2 months ago. She was receiving mycophenolate mofetil (MMF) $750 \mathrm{mg} /$ day, hydroxychloroquine (HCQ) $200 \mathrm{mg} /$ day, methylprednisolone $44 \mathrm{mg} /$ day, and irbesartan $150 \mathrm{mg} /$ day. Notably, the immunosuppressants were not discontinued. She was discharged as well after an 11-day hospital admission. Patient B was a 25-year-old female diagnosed with class III LN 1 year ago. She was taking MMF $1.5 \mathrm{~g} / \mathrm{day}$ and prednisone $15 \mathrm{mg} /$ day. She was not taking HCQ because of transient blurred vision half a year ago. MMF and prednisolone were temporarily held off on the day of diagnosis with COVID-19, and she was discharged as well after 14 days of hospitalization.

Nearly $60 \%$ of patients with LN had limited access to healthcare, and

Table 1. Medication usage prior to and during COVID-19 epidemic in 101 patients with lupus nephritis.

\begin{tabular}{lccc}
\hline Medication & $\begin{array}{c}\text { Prior to } \\
\text { COVID-19 }\end{array}$ & $\begin{array}{c}\text { Discontinued } \\
\text { during } \\
\text { COVID-19 }\end{array}$ & $\begin{array}{c}\text { Continued } \\
\text { during } \\
\text { COVID-19 }\end{array}$ \\
\hline Corticosteroids & $95(94)$ & $4(4)$ & $91(90)$ \\
Hydroxychloroquine & $52(51)$ & $8(8)$ & $44(44)$ \\
Mycophenolate mofetil & $17(17)$ & $4(4)$ & $13(13)$ \\
Cyclosporine/tacrolimus & $12(12)$ & $5(5)$ & $7(7)$ \\
Leflunomide & $2(2)$ & $0(0)$ & $2(2)$ \\
Azathioprine & $1(1)$ & $0(0)$ & $1(1)$ \\
Antihypertensive* & $25(25)$ & $5(5)$ & $20(20)$ \\
\hline
\end{tabular}

Data are $\mathrm{n}(\%)$. * Angiotensin-converting enzyme inhibitors and angiotensin II receptor blockers. COVID-19: coronavirus disease 2019. this resulted in a significant portion of patients discontinuing medications In this cohort, 2 patients contracted COVID-19 with mild disease. Patients with $\mathrm{LN}$ are threatened with interruption of continuity of care. Access to medical advice and care is needed for these vulnerable populations in the global COVID-19 pandemic. First, patients require guidance for immunosuppressant use because abrupt discontinuation may result in a flare of the disease. There is no evidence to suggest that immunosuppressed people are at higher risk of infection or develop more severe disease if they contract COVID-19 ${ }^{1}$. While the role of steroids is being debated ${ }^{2,3}$, certain rheumatology medications such as chloroquine, $\mathrm{HCQ}^{4,5}$, and baricitinib ${ }^{6}$ may have beneficial antiinflammatory and antiviral activity. Second, enforced quarantine in epidemic areas with overwhelmed healthcare resources will complicate clinic attendance. In the Department of Rheumatology, Allergy and Immunology at Tan Tock Seng Hospital in Singapore, outpatient appointments were massively reshuffled at the early stages of the COVID-19 outbreak in January 2020. The discontinuation of healthcare was therefore minimized. The care for patients with rheumatic disease should not be compromised in the era of the pandemic of COVID-19.

CHENG CHEN, MD, PhD, Department of Nephrology, Renmin Hospital of Wuhan University; BIQING YAO, MBBS, Department of Nephrology, Renmin Hospital of Wuhan University; MIAO YAN, MSc, Department of Nephrology, Renmin Hospital of Wuhan University; KE SU, MD, PhD, Department of Nephrology, Renmin Hospital of Wuhan University; HUIMING WANG, MD, PhD, Department of Nephrology, Renmin Hospital of Wuhan University, Wuhan, China; CHUANHUI XU, MD, PhD, Department of Rheumatology, Allergy, and Immunology, Tan Tock Seng Hospital, Singapore. Address correspondence to Dr. C. Xu, Tan Tock Seng Hospital, Department of Rheumatology, Allergy, and Immunology, Singapore.E-mail: xuchuanhui2008@gmail.com

\section{REFERENCES}

1. Wu Z, McGoogan JM. Characteristics of and important lessons from the coronavirus disease 2019 (COVID-19) outbreak in China: summary of a report of 72,314 cases from the Chinese Center for Disease Control and Prevention. JAMA 2020 Feb 24 (E-pub ahead of print).

2. Russell CD, Millar JE, Baillie JK. Clinical evidence does not support corticosteroid treatment for 2019-nCoV lung injury. Lancet 2020;395:473-5.

3. Shang L, Zhao J, Hu Y, Du R, Cao B. On the use of corticosteroids for 2019-nCoV pneumonia. Lancet 2020;395:683-4.

4. Yao X, Ye F, Zhang M, Cui C, Huang B, Niu P, et al. In vitro antiviral activity and projection of optimized dosing design of hydroxychloroquine for the treatment of severe acute respiratory syndrome coronavirus 2 (SARS-CoV-2). Clin Infect Dis 2020 Mar 9 (E-pub ahead of print).

5. Wang M, Cao R, Zhang L, Yang X, Liu J, Xu M, et al. Remdesivir and chloroquine effectively inhibit the recently emerged novel coronavirus (2019-nCoV) in vitro. Cell Res 2020;30:269-71.

6. Stebbing J, Phelan A, Griffin I, Tucker C, Oechsle O, Smith D, et al. COVID-19: combining antiviral and anti-inflammatory treatments. Lancet Infect Dis 2020;20:400-2.

First Release June 15, 2020; J Rheumatol 2020;47:9; doi: $10.3899 /$ jrheum.200452 